\title{
Functional acute acquired comitant esotropia: clinical characteristics and efficacy of single Botulinum toxin type $A$ injection
}

\author{
Luyao Tong ${ }^{1,2}$, Xiaoning Yu ${ }^{1}$, Xiajing Tang ${ }^{1}$, Yidong Zhang ${ }^{1}$, Sifan Zheng ${ }^{3}$ and Zhaohui Sun ${ }^{1 *}$
}

\begin{abstract}
Background: To examine the clinical features of acute acquired comitant esotropia (AACE) and to evaluate the clinical effectiveness of a single injection of botulinum toxin type A (BTXA) on binocular visual function (BVF).

Methods: This retrospective, observational case series study enrolled patients with AACE examined from October 2018-May 2019. BTXA was injected into the both medial rectus muscles. The refractive error, best-corrected visual acuity (BCVA), stereoacuity, vergence, accommodation, the horizontal angle of deviation, and the gradient accommodative convergence/accommodation (AC/A) ratio were measured pre- and post-BTXA injection. Data preand postinjection were compared by the Wilcoxon signed-rank test. A Spearman correlation coefficient was calculated to explore the relationships between demographic characteristics and BVF.

Results: Twenty-two AACE cases were included. Compared with preinjection deviation, the postinjection deviation in the primary position was smaller for near $(p<0.001)$ and distance $(p<0.001)$ fixation at 3 months after injection (BTXA). Furthermore, convergence was better for near $(p=0.003)$ and distance $(p<0.001)$ fixation, divergence was better for near $(p=0.021)$ and distance $(p<0.001)$ fixation, accommodation was better in the right $(p=0.011)$ and left $(p=0.004)$ eyes, and the gradient AC/A ratio was better at the third month after injection $(p=0.001)$. Stereoacuity was improved in 11 (50\%), unchanged in $5(22.73 \%)$ and decreased in 6 (27.27\%) patients. The preinjection stereoacuity $(p=0.013, r=0.522)$ and preinjection deviation for near $(p=0.015 r$, $=-0.512)$ and distance $(p=0.009, r=-0.541)$ were significantly associated with patient age.

Conclusions: AACE is characterized by a high AC/A ratio and low accommodation. A single injection of BTXA is effective for AACE. Deviation, stereoacuity, and the therapeutic effect of BTXA may be correlated with patient age.
\end{abstract}

Keywords: Acute acquired comitant esotropia, Botulinum toxin type a, Binocular visual function

\footnotetext{
* Correspondence: 2199010@zju.edu.cn

'Eye Center of the Second Affiliated Hospital, School of Medicine, Zhejiang University, Jiefang Road 88\#, Hangzhou 310009, Zhejiang Province, China

Full list of author information is available at the end of the article
}

(c) The Author(s). 2020 Open Access This article is licensed under a Creative Commons Attribution 4.0 International License, which permits use, sharing, adaptation, distribution and reproduction in any medium or format, as long as you give appropriate credit to the original author(s) and the source, provide a link to the Creative Commons licence, and indicate if changes were made. The images or other third party material in this article are included in the article's Creative Commons licence, unless indicated otherwise in a credit line to the material. If material is not included in the article's Creative Commons licence and your intended use is not permitted by statutory regulation or exceeds the permitted use, you will need to obtain permission directly from the copyright holder. To view a copy of this licence, visit http://creativecommons.org/licenses/by/4.0/. The Creative Commons Public Domain Dedication waiver (http://creativecommons.org/publicdomain/zero/1.0/) applies to the data made available in this article, unless otherwise stated in a credit line to the data. 


\section{Background}

Acute acquired comitant esotropia (AACE), a rare form of strabismus, describes sudden-onset constant esotropia with an equal angle of each gaze and leads to diplopia [1]. AACE was divided into the following 3 main types by Burian and Miller based on their different characteristics and etiologies [2]. Type 1 (Swan type) has sudden onset due to interrupted fusion (monocular occlusion or vision loss). Type 2 (Franceschetti type) may be caused by physical or mental stress (but not paralysis) and is characterized by large deviation, potential binocular vision, a low refractive error, and a minimal accommodative element. Type 3 (Bielschowsky type) is only relevant to the myopia of $-5.0 \mathrm{D}$ or more.

In increasing case reports, AACE has been reported to be related to the refractive error, decompensated esophoria, intracranial diseases (elevated intracranial pressure, brain glioma, thalamic or cerebellar tumor), and near work such as excessive smartphone use. However, these cases cannot be included in the Burian and Miller classification [3-10]. Some AACE cases were neuroimaging study-negative, systemic disease-negative, and without a history of neuro- or anesthesia-related medication use. Such AACE cases were associated only with refractive errors and binocular dysfunction, such as decompensated esophoria and high myopia, which were generally considered benign or functional [3, 4, 11, 12].

AACE causes uncomfortable diplopia and substantially affects the quality of life. Surgery, botulinum toxin type A (BTXA) injection, and wearing prism are current proposed as treatments for AACE. BTXA injection has the advantages of improving stereoacuity, maintaining ocular alignment, and reducing anesthesia and treatment costs in comparison with surgery [13-15]. BTXA injection has been shown to be effective for esotropia at a high rate. It is effective for several weeks, and is deemed to be an alternative treatment or an addition to traditional surgery [15-17]. However, no study has investigated the characteristics of binocular visual function (BVF) of AACE and changes in BVF after BTXA injections.

To explore the possible characteristics of BVF in functional AACE patients, we conducted this study to examine the clinical features of AACE without intracranial and neurological diseases and then evaluated the changes in BVF after a single injection of BTXA.

\section{Methods}

\section{Study population}

This retrospective study enrolled patients with AACE examined at the Eye Center of the Second Affiliated Hospital, School of Medicine, Zhejiang University, from 1st October 2018-31st May 2019.

The inclusion criteria were as follows: 1) sudden-onset constant esotropia with an equal angle of each gaze and diplopia, followed by a diagnosis of AACE; and 2) BTXA injection in the both medial rectus muscles.

The exclusion criteria were as follows: 1) a history of eye disease, monocular occlusion or vision loss, ocular surgery (except for refractive surgery), and ocular or head trauma; 2) systemic diseases, including intracranial disease, neurologic disease (all patients underwent a head computerized tomography examination and a neurological examination by neurologists before BTXA injection to rule out intracranial and neurological diseases), and diabetes mellitus; 3) a history of neuro- or anesthesia-related medication use; and 4) refusal to cooperate with a BVF examination or to comply with the follow-up.

\section{Measurements of deviation angle and binocular visual function}

We collected a complete medical history and personal details, including age, gender, the duration of diplopia, and the duration of daily near work from each patient. The following pre- and postinjection parameters were measured and recorded: refractive error, best-corrected visual acuity (BCVA), stereoacuity with the circles test of the Randot Stereotest (Stereo Optical Co., Inc.), the horizontal angle of deviation, vergence, accommodation, and the gradient accommodative convergence (prism diopter, PD)/accommodation (diopter, D) ratio (AC/A).

The horizontal angle of deviation was measured with the prism and cover tests at $33 \mathrm{~cm}$ (near) and $6 \mathrm{~m}$ (distance) fixation. After deviation was offset and diplopia was overlapped with a prism, convergence and divergence were re-examined with the prism and cover tests at near and distance fixation. This was measured by recording the patient's reports of a break to base-out and base-in prism. The vergence value was the sum of convergence and divergence. The accommodation was measured at near fixation with a minus lens. Patients were instructed to keep the target clear as the minus lens degree increased and to report when it first started to become defocused. When the target first started to become defocused, the value of the increased minus lens degree plus $3 \mathrm{D}$ was taken as a measure of accommodation. The $\mathrm{AC} / \mathrm{A}$ ratio was measured with the prism and cover tests at near fixation and determined by a deviation angle with additional pairs of lenses $(+1.00$ and -1.00 D) $[18,19]$. For statistical purposes, stereoacuity was converted to the reciprocal after measurement.

\section{BTXA injection method}

BTXA (Allergan, USA) injection into the both medial rectus muscle was performed after surface anesthesia of the conjunctiva by the same surgeon. The injection dose of BTXA was 3.5 units for patients with a deviation angle less than $25 \mathrm{PD}$ and 4.0 units for an angle greater than 
25 PD. To reduce leakage of BTXA, the surgeon fixed the target muscle with forceps, used a 30 gauge $\times 1 / 2$ in. $(0.3 \mathrm{~mm} \times 13 \mathrm{~mm})$ needle during injection, and held the needle for a few seconds before withdrawing it.

\section{Statistical analysis}

Statistical analyses were performed with SPSS version 21.0 (SPSS, Inc., Chicago, IL, USA). Data pre- and postinjection were compared by the Wilcoxon signed-rank test. Spearman's correlation coefficient was calculated to explore relationships between demographic characteristics and BVF. $P<0.05$ was considered statistically significant.

\section{Results}

Twenty-two AACE patients (13 males and 9 females) with a mean age of $26.32 \pm 7.19$ (range: $14-38$ ) years and sudden-onset, progressive, intermittent, or persistent horizontal binocular diplopia for 3 months to 10 years were included in the study. The mean duration of daily near work was $12.84 \pm 2.88$ (range: $8-18$ ) hours.

The mean spherical equivalent refractions were $4.35 \pm 2.18 \mathrm{D}$ in the right eye and $-4.14 \pm 2.26 \mathrm{D}$ in the left eye. Each patient with refractive error wore fullcorrective glasses before and after BTXA injection for a large proportion of the day, including work, study, and daily life. Binocular BCVA was 20/20 in all patients except one, whose binocular BCVA was 18/20. Eye movements in all directions were free. Ocular examinations and neurological examinations were normal in all patients. The follow-up duration was 3 to 4 months. Clinical characteristics can be found in the Table 1.

Compared with preinjection deviation, the postinjection deviation in the primary position was smaller both for near $(p<0.001)$ and distance $(p<0.001)$ fixation at 3 months after injection. The divergence and convergence were better for both near and distance fixation. The accommodation was better in both eyes. The gradient $\mathrm{AC} /$ A ratio was better in the third month (Table 2). Stereoacuity was improved in 11 (50\%), unchanged in 5 $(22.73 \%)$ and decreased in $6(27.27 \%)$ patients. All 7 patients without stereoacuity achieved stereoscopic vision after injection.

Immediately following BTXA injection, diplopia disappeared in all patients. In the third month of follow-up, diplopia had disappeared or had appeared occasionally in $15(68.2 \%)$ patients. 7 (31.8\%) patients had persistent diplopia. None of the patients had complications such as infection, bleeding, ptosis, or restricted eye movement. No patients experienced overcorrection.

The Spearman's correlation was calculated on demographic characteristics, deviation and BVF. The preinjection stereoacuity was not significantly associated with deviation for near $(p=0.066, r=-0.399)$ and distance $(p=0.270, r=-0.224)$, accommodation in the right eye $(p=0.267, r=0.247)$ and left eye $(p=0.683, r=0.092)$, and the duration of daily near work $(p=0.980, r=$ $0.006)$, but was associated with patient age $(p=0.013$, $r=0.522)$. Preinjection deviations for near $(p=0.015, r=$ $-0.512)$ and distance $(p=0.009, r=-0.541)$, and reductions in deviations after injection for near $(p=0.003, r=$ $0.601)$ and distance $(p<0.001, r=0.699)$ were significantly associated with patient age. However, the duration of daily near work was not significantly associated with any BVF measured in this study.

\section{Discussion}

The study showed that AACE was characterized by a high $\mathrm{AC} / \mathrm{A}$ and low accommodation. A single injection of BTXA was effective for AACE. These findings may provide a reference for subsequent treatment.

AACE has long been divided into 3 main types based on the characteristics of interrupted fusion, physical or mental stress, and mildly hypermetropia [2]. However, some reported cases did not fit into these types $[3-5,7$, $8,12]$. According to imaging evidence, neurological and intracranial diseases are undoubtedly the causes of AACE $[5,6,9,10]$. In this study, the AACE patients did not suffer from intracranial or neurological diseases. Some patients suffered from high myopia or esotropia with a small angle. Without a history of eye disease, monocular occlusion or vision loss, ocular surgery, or ocular trauma, the patients in this study did not meet the classification. A new classification should be proposed to cover these cases. Therefore, functional or benign causes of AACE should be considered, and BVF should be examined.

After excluding diseases of the brain, extraocular muscles, and orbit, Ali et al. included 8 cases of progressive intermittent horizontal esotropia and speculated that decompensated esophoria was a benign cause of AACE [4]. However, their study did not study for AC/A ratio. The $\mathrm{AC} / \mathrm{A}$ ratio shows some characteristics in comitant strabismus and changes after treatment. Bateman and Parks found that the AC/A was normal, high, and low in 62, 34 , and $4 \%$ of concomitant esotropia patients, respectively. AC/A ratio was closer to normal after surgery [20]. A prospective study by Lucas et al. found the mean $\mathrm{AC} /$ A of 25 patients with comitant esotropia decreased from 8 to 4 [21]. In this study, the mean AC/A ratio of 22 patients was higher than those in normal (between 3 and 5 $\mathrm{PD} / \mathrm{D}$ ) [19] and esotropic (an average of $3.15 \mathrm{PD} / \mathrm{D}$ ) populations [22], and accommodation was lower than age-matched values [23]. The AC/A ratio was also found to be high in convergence excess cases [24], suggesting that convergence excess may be a characteristic of AACE, which produces more convergence with the same accommodation requirements [19]. The lack of accommodative 


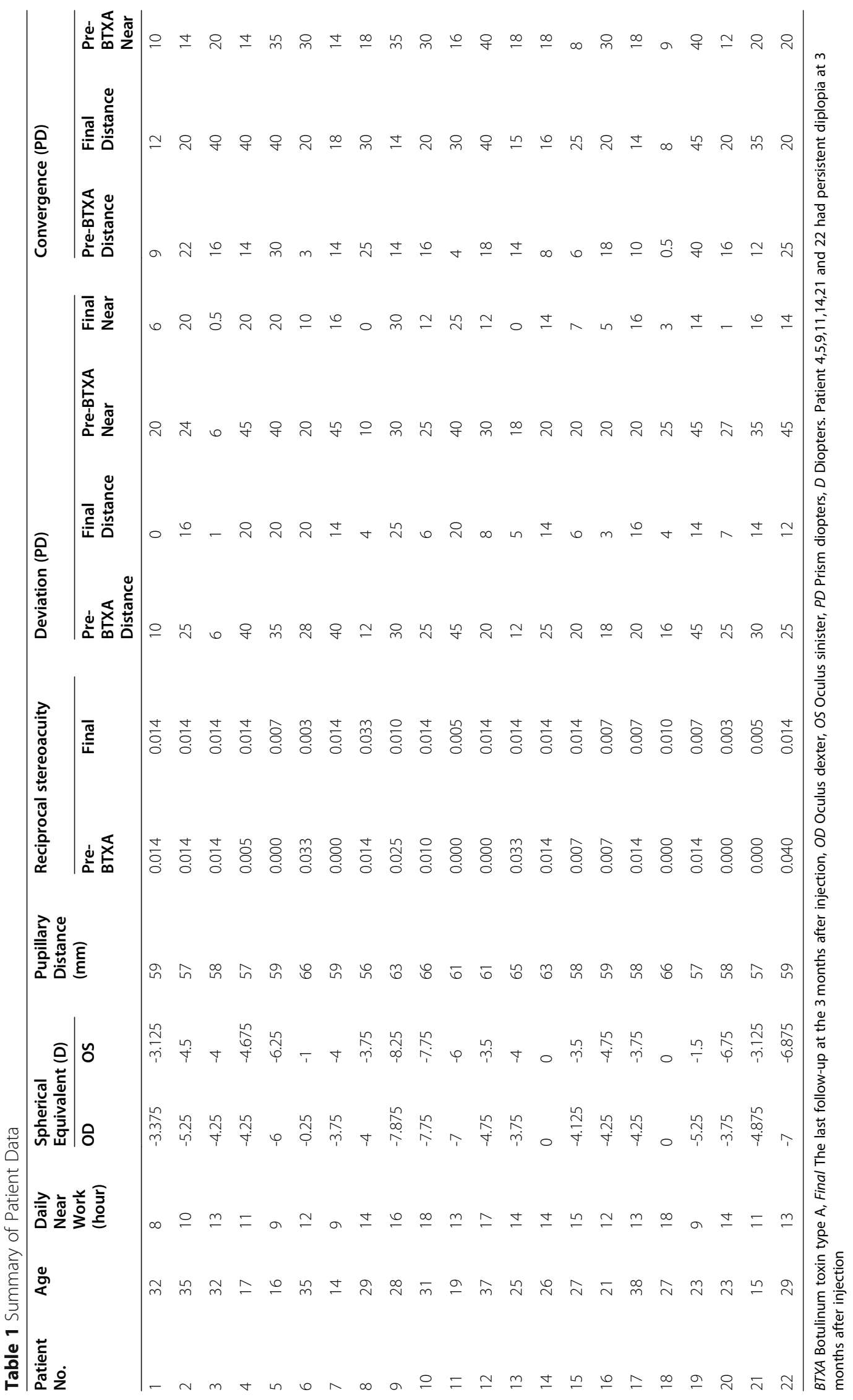




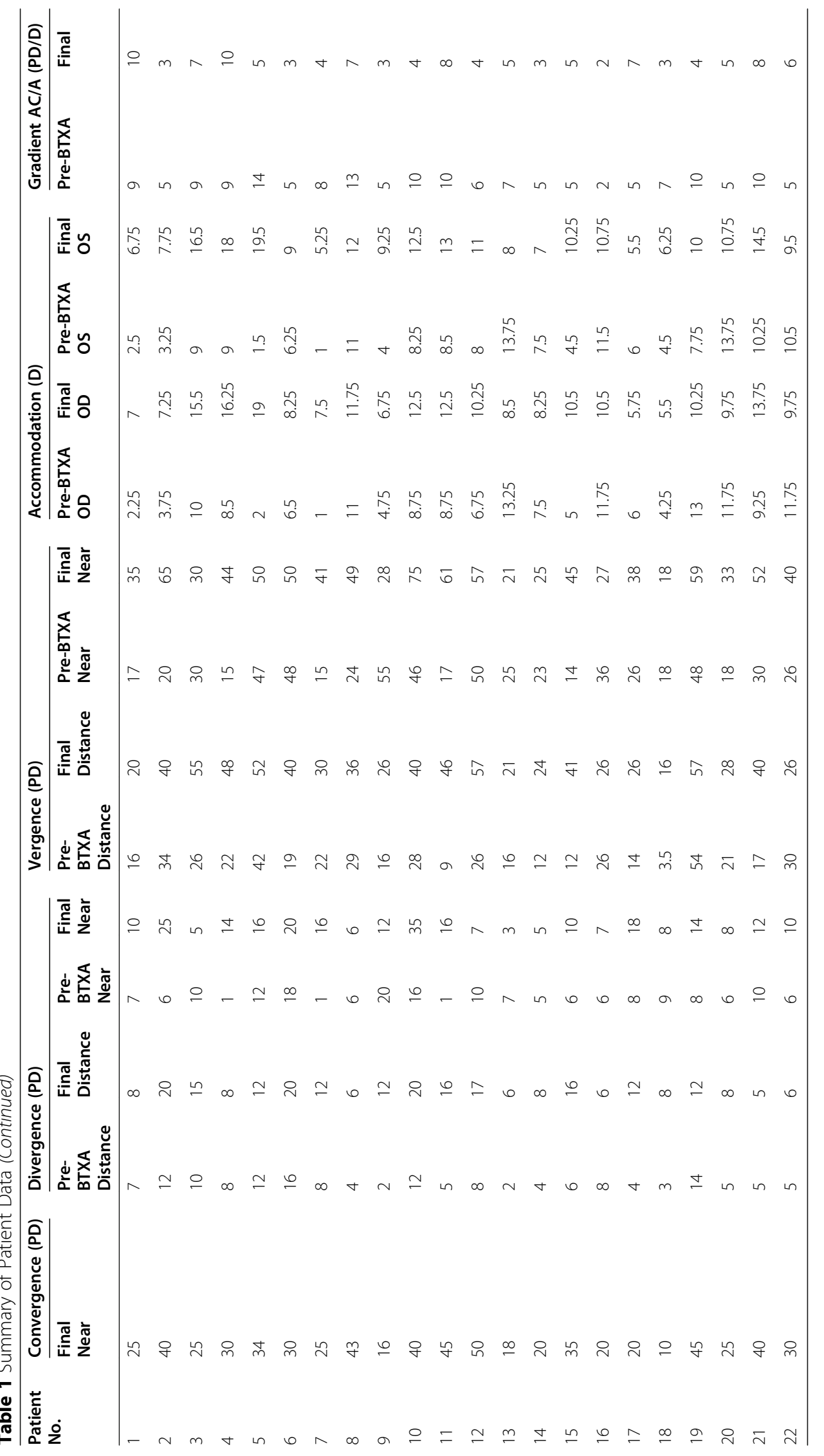


Table 2 Clinical characteristics of patients pre- and post-BTXA injection

\begin{tabular}{|c|c|c|c|c|c|c|}
\hline \multirow{2}{*}{$\begin{array}{l}\text { Characteristics } \\
n=22\end{array}$} & & \multicolumn{4}{|c|}{ Mean \pm 1 Standard deviation (Range) } & \multirow{3}{*}{$\begin{array}{l}\boldsymbol{P} \text { Value } \\
0.887\end{array}$} \\
\hline & & \multicolumn{2}{|c|}{ Pre-BTXA injection } & \multicolumn{2}{|c|}{ Post-BTXA injection } & \\
\hline Reciprocal stereoacuity & & $0.012 \pm 0.012$ & $(0.000-0.040)$ & $0.012 \pm 0.007$ & $(0.003-0.033)$ & \\
\hline \multirow[t]{2}{*}{ Deviation (PD) } & Distance & $25.09 \pm 10.99$ & $(6.0-45.0)$ & $11.32 \pm 7.23 \mathrm{PD}$ & $(0.0-25.0)$ & $<0.001$ \\
\hline & Near & $27.73 \pm 11.60$ & $(6.0-45.0)$ & $11.89 \pm 8.40$ & $(0.0-30.0)$ & $<0.001$ \\
\hline \multirow[t]{2}{*}{ Convergence (PD) } & Distance & $15.20 \pm 9.25$ & $(0.5-40.0)$ & $24.64 \pm 10.97$ & $(8.0-45.0)$ & $<0.001$ \\
\hline & Near & $21.32 \pm 9.95$ & $(8.0-40.0)$ & $30.27 \pm 10.91$ & $(10.0-50.0)$ & 0.003 \\
\hline \multirow[t]{2}{*}{ Divergence (PD) } & Distance & $7.24 \pm 3.94$ & $(2.0-16.0)$ & $11.50 \pm 5.00$ & $(5.0-20.0)$ & $<0.001$ \\
\hline & Near & $8.14 \pm 4.97$ & $(1.0-20.0)$ & $12.59 \pm 7.42$ & $(3.0-35.0)$ & 0.021 \\
\hline \multirow[t]{2}{*}{ Vergence (PD) } & Distance & $22.48 \pm 11.29$ & $(3.5-54.0)$ & $36.14 \pm 12.64$ & $(16.0-57.0)$ & $<0.001$ \\
\hline & Near & $29.45 \pm 13.49$ & $(14.0-55.0)$ & $42.86 \pm 15.10$ & $(18.0-75.0)$ & 0.003 \\
\hline \multirow[t]{2}{*}{ Accommodation (D) } & OD & $7.61 \pm 3.69$ & $(1.00-13.25)$ & $10.32 \pm 3.51$ & $(5.00-19.00)$ & 0.011 \\
\hline & OS & $7.38 \pm 3.68$ & $(1.00-13.75)$ & $10.59 \pm 3.89$ & $(5.25-19.50)$ & 0.004 \\
\hline Gradient AC/A (PD/D) & & $7.45 \pm 2.99$ & $(2.0-14.0)$ & $5.27 \pm 2.31$ & $(2.0-10.0)$ & 0.001 \\
\hline
\end{tabular}

$B T X A$ Botulinum toxin type $A$, Final the last follow-up at the 3 months after injection, $O D$ Oculus dexter, OS Oculus sinister, $P D$ Prism diopters, $D$ Diopters

ability leads to more convergent accommodation when patients view objects at close range. This induces chronic overuse of the both medial rectus muscles in patients with AACE, which may strengthen the medial rectus muscles and break the balance of the medial and lateral rectus muscles. This may subsequently be the cause of AACE in patients using smartphones at close proximity for a long time $[7,8]$.

Refractive error may be another cause of AACE. In a study by Simon and Borchert [3], after correcting for hypermetropia, esotropia improved in seven patients. The study speculated that an uncorrected refractive error may be the cause of acute late onset esotropia. As each patient with refractive error wore full-corrective glasses for most of their daily lives in our study, we found that preinjection deviation for near and distance was not associated with the spherical equivalent in each eye. This may partly support Simon and Borchert's view and suggest that correction of refractive errors is not correlated with the incidence of AACE. This hypothesis should be tested in a controlled clinical trial.

BTXA is a neurotoxin that selectively blocks the release of acetylcholine at the neuromuscular junction and inhibits muscle contraction [25]. Therefore, BTXA affects BVF associated with the injected extraocular muscles. However, few studies have described BVF changes postinjection. After a single BTXA injection in this study, the deviation of the AACE patients decreased. The convergence, divergence, and accommodation improved. The post-injection AC/A ratio in this study decreased and was close to normal, which may suggest that BTXA can relieve excessive convergence by inhibiting contraction of the medial rectus muscle. The accommodation in our study was reflex accommodation induced by a blurred retinal image with a minus lens.
We could not find conclusive evidence to support the effect of a high $\mathrm{AC} / \mathrm{A}$ ratio or excessive convergence on reflex accommodation in existing studies. However, we can speculate that the increase in convergence accommodation may lead to a decrease in reflex accommodation, when the amplitude of accommodation is fixed. Accommodation improved at 3 months after the injection, which may have been due to remission of convergence accommodation after deviation was alleviated.

Compared with globe perforation, slipped muscle, scleritis, and intraoperative muscle loss, BTXA is less invasive, muscle-sparing [26], and the injection repeatable up to 9 times with no significant side effects [15]. Without the need for incision or suturing of the extraocular muscles and scar formation, patients may achieve closer to normal BVF after BTXA injection. Based on this speculation, we will set up a surgery group and a BTXA injection group for comparison in further study.

BVF change with age. Older patients $(40-70$ years of age) had a more tonic convergence position than younger patients (20-39 years of age) [27]. The magnitude of phoria adaptation and the base-in and base-out recovery decreased with age $[28,29]$. The AC/A ratio increases with age, which may be associated with tonic accommodation and vergence systems [30]. Therefore, we also considered the effect of age on the visual factors of AACE. Research on the epidemiology of AACE is currently lacking. In existing studies and case reports, most AACE patients are children and young adults. $\mathrm{Fu}$ et al. found that the mean deviation of esotropia in younger children was significantly larger than that in older children and adults in the Chinese population. A younger onset age may be a characteristic of AACE and may also be associated with esotropia deviation [11]. In our study, the younger patients experienced worse preinjection 
stereopsis and larger preinjection deviations for near and distance. We suspect that the stronger esophoria adaptation and recovery of younger patients delayed the onset of diplopia, leading to a more advanced stage of AACE and a greater deviation angle. The reductions in deviations for near and distance in this study were greater in older patients, possibly because the BTXA injection attenuated more tonic convergence in older patients when inhibiting muscle contraction.

Uncorrected strabismus or the presence of diplopia after treatment is defined as recurrence. Clark et al. reported a $1 / 6(16.7 \%)$ recurrence rate for AACE treated by surgery [1]. Dawson et al. reported that 2/14 (14.3\%) patients had unstable ocular positions after toxin injection [13]. In a study conducted by Wan et al., the success rates of BTXA injections were $81 \%$ at 6 months and $67 \%$ at 18 months, compared with the surgery group, where the success rates were $61 \%$ at 6 months and $58 \%$ at 18 months [14]. Conversely, Alejandra et al. reported a treatment success rate of $66 \%$ of surgical procedures compared with $45 \%$ of BTXA injections [16]. In this study, although deviation was large in some patients, they had similar or greater divergent functions numerically. These patients experienced diplopia only in the morning or with severe eye strain. In contrast, some patients had less deviation but substantially less divergence, with persistent diplopia after the injection. Before this study, AACE patients showed symptoms of overcorrection after BXTA injection. We gradually adjusted the dose and determined the current injection dose of 3.5-4 units. In this study, no patients experienced overcorrection.

Since the dose of BTXA cannot be accurately measured according to the angle of deviation and the main objective of AACE treatment is diplopia relief, BTXA treatment of AACE can be considered satisfactory under the following conditions: 1) divergence ability after treatment can compensate for strabismus; 2) diplopia symptoms do not appear in daily life; 3 ) a satisfactory quality of life can be achieved. In our study, diplopia disappeared or appeared only briefly in 15 (68.2\%) patients after a single BTXA injection, reflecting a satisfactory therapeutic effect. Patients with recurrence can still be reinjected with BTXA or treated with surgery.

Some limitations may exist in this study. AACE patients were in a state of binocular diplopia and esotropia when they visited the doctor. BVF before onset was unknown and difficult to assess. Secondly, the dominant eye may have switched due to diplopia and BVF changed. The possible influence of dominant eye function on AACE was difficult to investigate. Finally, the follow-up period was short, and the long-term recurrence of AACE after a single BTXA injection was unknown.

\section{Conclusion}

AACE without intracranial and neurological diseases is characterized by a high AC/A and low accommodation. A single injection of BTXA is effective for AACE. Patient age may be an important factor correlated with deviation, stereoacuity, and the therapeutic effect of BTXA.

\section{Abbreviations}

AACE: Acute acquired comitant esotropia; BTXA: Botulinum toxin type $A$; BVF: Binocular visual function; D: Diopter; PD: Prism diopter; BCVA: Bestcorrected visual acuity; AC/A: Accommodative convergence/accommodation ratio

\section{Acknowledgments}

Not applicable.

\section{Authors' contributions}

All authors conceived and designed the study protocol. ZS, XT, and YZ provided patient care. ZS performed the surgery. LT collected the data. XY and LT were involved in the analysis and wrote the first draft of the manuscript. ZS and SZ reviewed and revised the manuscript and produced the final version. All authors read and approved the final manuscript.

\section{Funding}

Supported by the National Natural Science Foundation of China (No.81800869). The funder was involved in the analysis and wrote the first draft of the manuscript.

\section{Availability of data and materials}

The datasets used and analyzed during the current study are available from the corresponding author on reasonable request.

\section{Ethics approval and consent to participate}

This retrospective case series study and acquisition of data were approved by the Institutional Review Board of the Second Affiliated Hospital, School of Medicine, Zhejiang University in Hangzhou, China, and conducted under the principles of the Declaration of Helsinki. The data used in this study was anonymized before its use.

\section{Consent for publication}

Not applicable.

\section{Competing interests}

No competing interests.

\section{Author details}

${ }^{1}$ Eye Center of the Second Affiliated Hospital, School of Medicine, Zhejiang University, Jiefang Road 88\#, Hangzhou 310009, Zhejiang Province, China. ${ }^{2}$ Affiliated Hospital of Medical School of Ningbo University, Ningbo, Zhejiang Province, China. ${ }^{3}$ GKT School of Medical Education, King's College London, London, SE1 1UL, England.

Received: 1 May 2020 Accepted: 17 November 2020

Published online: 25 November 2020

\section{References}

1. Clark AC, Nelson LB, Simon JW, Wagner R, Rubin SE. Acute acquired comitant esotropia. Br J Ophthalmol. 1989;73(8):636-8.

2. Burian $\mathrm{HM}$, Miller JE. Comitant convergent strabismus with acute onset. Am J Ophthalmol. 1958;45(4):55-64

3. Simon AL, Borchert M. Etiology and prognosis of acute, late-onset Esotropia. Ophthalmology. 1997;104(8):1348-52.

4. Ali MH, Berry S, Qureshi A, Rattanalert N, Demer JL. Decompensated Esophoria as a benign cause of acquired Esotropia. Am J Ophthalmol. 2018; 194:95-100.

5. Buch $H$, Vinding T. Acute acquired comitant esotropia of childhood: a classification based on 48 children. Acta Ophthalmol. 2015;93(6):568-74.

6. Williams AS, Hoyt CS. Acute comitant esotropia in children with brain tumors. Arch Ophthalmol. 1989;107(3):376-8. 
7. Mehta A, Greensher JE, Dahl GJ, Miller KE. Acute onset Esotropia from excessive smartphone use in a teenager. J Pediatr Ophthalmol Strabismus. 2018;55:e42-4

8. Lee HS, Park SW, Heo H. Acute acquired comitant esotropia related to excessive smartphone use. BMC Ophthalmol. 2016;16:37.

9. Lee JM, Kim SH, Lee JI, Ryou JY, Kim SY. Acute comitant esotropia in a child with a cerebellar tumor. Korean J Ophthalmol. 2009;23(3):228-31.

10. Parentin F, Marchetti F, Faleschini E, Tonini G, Pensiero S. Acute comitant esotropia secondary to idiopathic intracranial hypertension in a child receiving recombinant human growth hormone. Can J Ophthalmol J Can D'Ophtalmol. 2009:44(1):110-1.

11. Fu T, Wang J, Levin M, Xi P, Li D, Li J. Clinical features of acute acquired comitant esotropia in the Chinese populations. Medicine. 2017;96(46):e8528.

12. Spierer A. Acute concomitant esotropia of adulthood. Ophthalmology. 2003; 110(5):1053-6.

13. Dawson ELM, Marshman WE, Adams GGW. The role of botulinum toxin a in acute-onset esotropia. Ophthalmology. 1999;106(9):1727-30

14. Wan MJ, Mantagos IS, Shah AS, Kazlas M, Hunter DG. Comparison of Botulinum toxin with surgery for the treatment of acute-onset Comitant Esotropia in children. Am J Ophthalmol. 2017;176:33-9.

15. Scott AB. Botulinum toxin injection into Extraocular muscles as an alternative to strabismus surgery. Ophthalmology. 1980:87(10):1044-9.

16. de Alba Campomanes AG, Binenbaum G, Campomanes Eguiarte G. Comparison of botulinum toxin with surgery as primary treatment for infantile esotropia. J AAPOS. 2010;14(2):111-6.

17. Lueder GT, Galli M, Tychsen L, Yildirim C, Pegado V. Long-term results of botulinum toxin-augmented medial rectus recessions for large-angle infantile esotropia. Am J Ophthalmol. 2012;153(3):560-3.

18. Brautaset RL, Jennings AJ. Effects of orthoptic treatment on the $C A / C$ and AC/a ratios in convergence insufficiency. Invest Ophthalmol Vis Sci. 2006; 47(7):2876-80.

19. Hughes A. AC/a ratio. Br J Ophthalmol. 1967;51(11):786-7.

20. Bateman JB, Parks MM. Clinical and computer-assisted analyses of preoperative and postoperative accommodative convergence and accommodation relationships. Ophthalmology. 1981;88(10):1024-30.

21. Lucas E, Bentley $C R$, Aclimandos WA. The effect of surgery on the AC/a ratio. Eye. 1994;8(Pt 1):109-14.

22. Franceschetti AT, Burian HM. Gradient accommodative convergence/ accommodative ratio in families with and without Esotropia. Am J Ophthalmol. 1970;70(4):558-62.

23. Duane A. Normal values of the accommodation at all ages. J Am Med Assoc. 1912;12:1010

24. Nilsson M, Brautaset RL. Vergence adaptation in subjects with convergence excess. Strabismus. 2011:19(1):5-11.

25. Turton K, Chaddock JA, Acharya KR. Botulinum and tetanus neurotoxins: structure, function and therapeutic utility. Trends Biochem Sci. 2002;27(11): 552-8.

26. Bradbury JA, Taylor RH. Severe complications of strabismus surgery. J AAPOS. 2013;17(1):59-63.

27. Ciuffreda KJ, Ong E, Rosenfield M. Tonic vergence, age and clinical presbyopia. Ophthalmic Physiol Opt. 1993;13(3):313-5.

28. Alvarez TL, Kim EH, Yaramothu C, Granger-Donetti B. The influence of age on adaptation of disparity vergence and phoria. Vis Res. 2017;133:1-11.

29. Palomo Alvarez C, Puell MC, Sanchez-Ramos C, Villena C. Normal values of distance heterophoria and fusional vergence ranges and effects of age. Graefes Arch Clin Exp Ophthalmol. 2006;244(7):821-4.

30. Bruce AS, Atchison DA, Bhoola H. Accommodation-convergence relationships and age. Invest Ophthalmol Vis Sci. 1995;36(2):406-13.

\section{Publisher's Note}

Springer Nature remains neutral with regard to jurisdictional claims in published maps and institutional affiliations.

Ready to submit your research? Choose BMC and benefit from:

- fast, convenient online submission

- thorough peer review by experienced researchers in your field

- rapid publication on acceptance

- support for research data, including large and complex data types

- gold Open Access which fosters wider collaboration and increased citations

- maximum visibility for your research: over $100 \mathrm{M}$ website views per year

At $\mathrm{BMC}$, research is always in progress.

Learn more biomedcentral.com/submissions 